\author{
OAK RIDGE \\ NATIONAL LABORATORY
}

MANAGED BY UT-BATTELLE

FOR THE DEPARTMENT OF ENERGY

\title{
Data Compilation for AGR-2 UCO Variant Coated Particle Batch G73J-14-93073A
}

John D. Hunn

October 2008

This report was prepared as an account of work sponsored by an agency of the United States Government. Neither the United States Government nor any agency thereof, or any of their employees, makes any warranty, expressed or implied, or assumes any legal liability or responsibility for any third party's use, or the results of such use, of any information, apparatus, product or process disclosed in this report, or represents that its use by such third party would not infringe privately owned rights. 


\section{DOCUMENT AVAILABILITY}

Reports produced after January 1, 1996, are generally available free via the U.S. Department of Energy (DOE) Information Bridge.

Web site http://www.osti.gov/bridge

Reports produced before January 1, 1996, may be purchased by members of the public from the following source.

National Technical Information Service

5285 Port Royal Road

Springfield, VA 22161

Telephone 703-605-6000 (1-800-553-6847)

TDD 703-487-4639

Fax 703-605-6900

E-mail info@ntis.fedworld.gov

Web site http://www.ntis.gov/support/ordernowabout.htm

Reports are available to DOE employees, DOE contractors, Energy Technology Data Exchange (ETDE) representatives, and International Nuclear Information System (INIS)

representatives from the following source.

Office of Scientific and Technical Information

P.O. Box 62

Oak Ridge, TN 37831

Telephone 865-576-8401

Fax 865-576-5728

E-mail reports@adonis.osti.gov

Web site http://www.osti.gov/contact.html

This report was prepared as an account of work sponsored by an agency of the United States Government. Neither the United States Government nor any agency thereof, nor any of their employees, makes any warranty, express or implied, or assumes any legal liability or responsibility for the accuracy, completeness, or usefulness of any information, apparatus, product, or process disclosed, or represents that its use would not infringe privately owned rights. Reference herein to any specific commercial product, process, or service by trade name, trademark, manufacturer, or otherwise, does not necessarily constitute or imply its endorsement, recommendation, or favoring by the United States Government or any agency thereof. The views and opinions of authors expressed herein do not necessarily state or reflect those of the United States Government or any agency thereof. 


\title{
Data Compilation for AGR-2 UCO Variant Coated Particle Batch G73J-14-93073A
}

\author{
John D. Hunn \\ Oak Ridge National Laboratory
}

Coated particle fuel batch G73J-14-93073A was produced by the Babcock and Wilcox Company $(\mathrm{B} \& \mathrm{~W})$ for possible selection as UCO variant fuel for the Advanced Gas Reactor Fuel Development and Qualification Program's AGR-2 irradiation test. Batch G73J-14-93073A is a single batch of TRISO-coated $425 \mu \mathrm{m}$ nominal diameter $14 \%$ low enrichment uranium oxide/uranium carbide kernels (LEUCO). The TRISO-coatings consist of a 50\% dense carbon buffer layer (100 $\mu \mathrm{m}$ nominal thickness) followed by a dense inner pyrocarbon layer (40 $\mu \mathrm{m}$ nominal thickness) followed by a SiC layer (35 $\mu$ m nominal thickness) followed by another dense outer pyrocarbon layer ( $40 \mu \mathrm{m}$ nominal thickness). For this variant, argon was added to the hydrogen fluidization gas during $\mathrm{SiC}$ deposition.

The AGR-2 Fuel Specification (INL SPC-923) provides the requirements necessary for acceptance of the fuel manufactured for the AGR-2 irradiation test. The bulk of the kernels and coated particle acceptance testing was performed at $\mathrm{B} \& \mathrm{~W}$ and is not contained in this report. Sample NP-B7958 was sent to ORNL for supplemental characterization. The procedures for the limited characterization and qualification of the particles performed at ORNL are outlined in ORNL product inspection plan AGR-CHAR-PIP-09. The BAFo equivalent optical anisotropies of the inner and outer pyrocarbon layers are reported on Inspection Report Form IRF-09, with a determination as to whether the particle batch satisfied the specified parameters for this property. The batch was found to satisfy the AGR-2 Fuel Specification SPC-923, Rev. 1 for IPyC and OPyC anisotropy.

Also provided in this data package are data on the true BAFo, average particle weight, OPyC open porosity, and $\mathrm{SiC}$ soot inclusion defect fraction. True BAFo is calculated as $(1+\mathrm{N}) /(1-\mathrm{N})$, where $\mathrm{N}$ is the diattenuation. This differs from equivalent $\mathrm{BAFo}=1+3 \mathrm{~N}$, which is the calculation used by the fuel specification to allow comparison to historical measurements. Average $\mathrm{OPyC}$ open porosity was determined using a single sample to be $0.41 \mathrm{ml} / \mathrm{m}^{2}$. One possible $\mathrm{SiC}$ soot inclusion was found in a sample of 4729 particles. This corresponds to $<1 \mathrm{E}-3$ defect fraction at $95 \%$ confidence. 


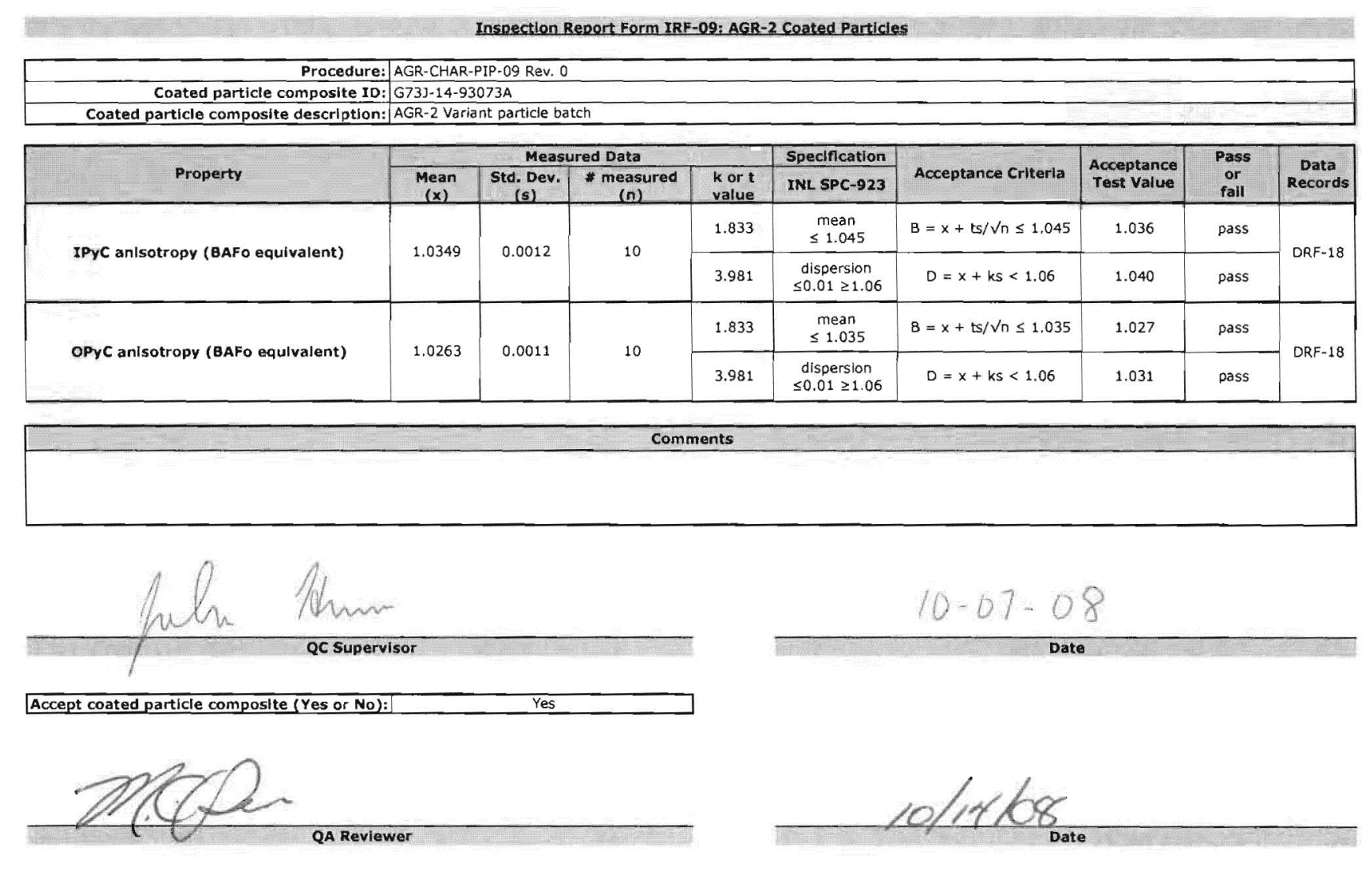


Data Report Form DRF-18A: Measurement of Pyrocarbon Anisotropy using the 2-MGEM - IPYC

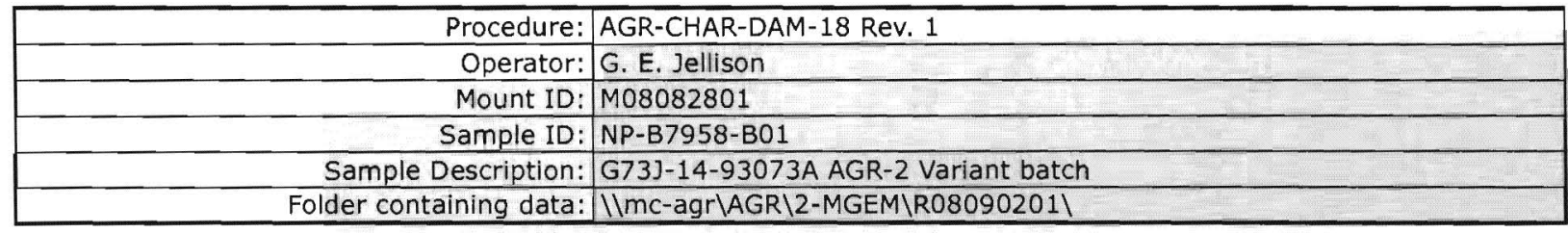

\begin{tabular}{|c|c|c|c|c|c|c|c|}
\hline \multirow{2}{*}{ Particle \# } & \multirow{2}{*}{$\begin{array}{c}\text { Grid } \\
\text { Position }\end{array}$} & \multicolumn{3}{|c|}{ Diattenuation } & \multicolumn{3}{|c|}{ Equivalent $\mathrm{BAFO}=1+3 \mathrm{~N}$} \\
\hline & & Average & St. Dev. & Ave. Error & Average & St. Dev. & Ave. Error \\
\hline 1 & 4,4 & 0.0122 & 0.0018 & 0.0005 & 1.0366 & 0.0054 & 0.0015 \\
\hline 2 & 4,5 & 0.0117 & 0.0018 & 0.0005 & 1.0351 & 0.0054 & 0.0015 \\
\hline 3 & 4,6 & 0.0113 & 0.0021 & 0.0005 & 1.0339 & 0.0063 & 0.0015 \\
\hline 4 & 5,4 & 0.0116 & 0.0020 & 0.0005 & 1.0348 & 0.0060 & 0.0015 \\
\hline 5 & 5,5 & 0.0116 & 0.0021 & 0.0005 & 1.0348 & 0.0063 & 0.0015 \\
\hline 6 & 5,6 & 0.0112 & 0.0021 & 0.0005 & 1.0336 & 0.0063 & 0.0015 \\
\hline 7 & 6,4 & 0.0121 & 0.0020 & 0.0005 & 1.0363 & 0.0060 & 0.0015 \\
\hline 8 & 6,5 & 0.0118 & 0.0020 & 0.0005 & 1.0354 & 0.0060 & 0.0015 \\
\hline 9 & 6,6 & 0.0120 & 0.0018 & 0.0005 & 1.0360 & 0.0054 & 0.0015 \\
\hline 10 & 5,7 & 0.0109 & 0.0017 & 0.0005 & 1.0327 & 0.0051 & 0.0015 \\
\hline \multicolumn{2}{|c|}{ Average } & 0.0116 & 0.0019 & 0.0005 & 1.0349 & 0.0058 & 0.0015 \\
\hline
\end{tabular}

Mean of average BAFo per particle: 1.0349

Standard deviation of average BAFo per particle: 0.0012

\section{Comments}
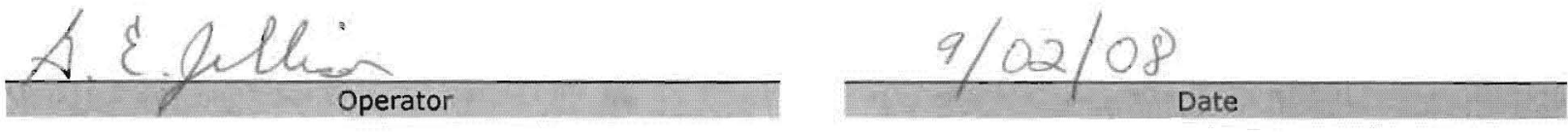


\section{Data Report Form DRF-18B: Measurement of Pyrocarbon Anisotropy using the 2-MGEM - OPYC}

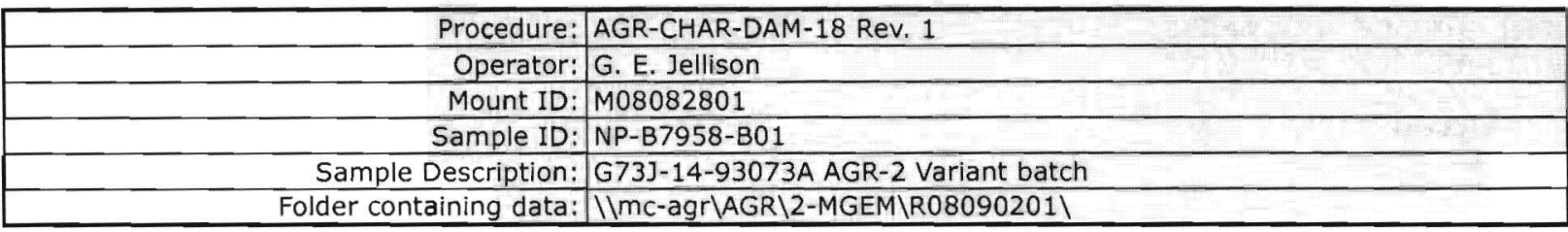

\begin{tabular}{|c|c|c|c|c|c|c|c|}
\hline \multirow{2}{*}{ Particle \# } & \multirow{2}{*}{$\begin{array}{c}\text { Grid } \\
\text { Position }\end{array}$} & \multicolumn{3}{|c|}{ Diattenuation } & \multicolumn{3}{|c|}{ Equivalent BAFo $=1+3 \mathrm{~N}$} \\
\hline & & Average & St. Dev. & Ave. Error & Average & St. Dev. & Ave. Error \\
\hline 1 & 4,4 & 0.0089 & 0.0016 & 0.0005 & 1.0267 & 0.0048 & 0.0015 \\
\hline 2 & 4,5 & 0.0090 & 0.0017 & 0.0005 & 1.0270 & 0.0051 & 0.0015 \\
\hline 3 & 4,6 & 0.0089 & 0.0018 & 0.0005 & 1.0267 & 0.0054 & 0.0015 \\
\hline 4 & 5,4 & 0.0092 & 0.0017 & 0.0005 & 1.0276 & 0.0051 & 0.0015 \\
\hline 5 & 5,5 & 0.0091 & 0.0020 & 0.0005 & 1.0273 & 0.0060 & 0.0015 \\
\hline 6 & 5,6 & 0.0082 & 0.0019 & 0.0005 & 1.0246 & 0.0057 & 0.0015 \\
\hline 7 & 6,4 & 0.0083 & 0.0016 & 0.0005 & 1.0249 & 0.0048 & 0.0015 \\
\hline 8 & 6,5 & 0.0082 & 0.0018 & 0.0005 & 1.0246 & 0.0054 & 0.0015 \\
\hline 9 & 6,6 & 0.0089 & 0.0018 & 0.0005 & 1.0267 & 0.0054 & 0.0015 \\
\hline 10 & 5,7 & 0.0089 & 0.0017 & 0.0005 & 1.0267 & 0.0051 & 0.0015 \\
\hline \multicolumn{2}{|c|}{ Average } & 0.0088 & 0.0018 & 0.0005 & 1.0263 & 0.0053 & 0.0015 \\
\hline
\end{tabular}

Mean of average BAFo per particle: 1.0263 Standard deviation of average BAFo per particle: 0.0011

\section{Comments}

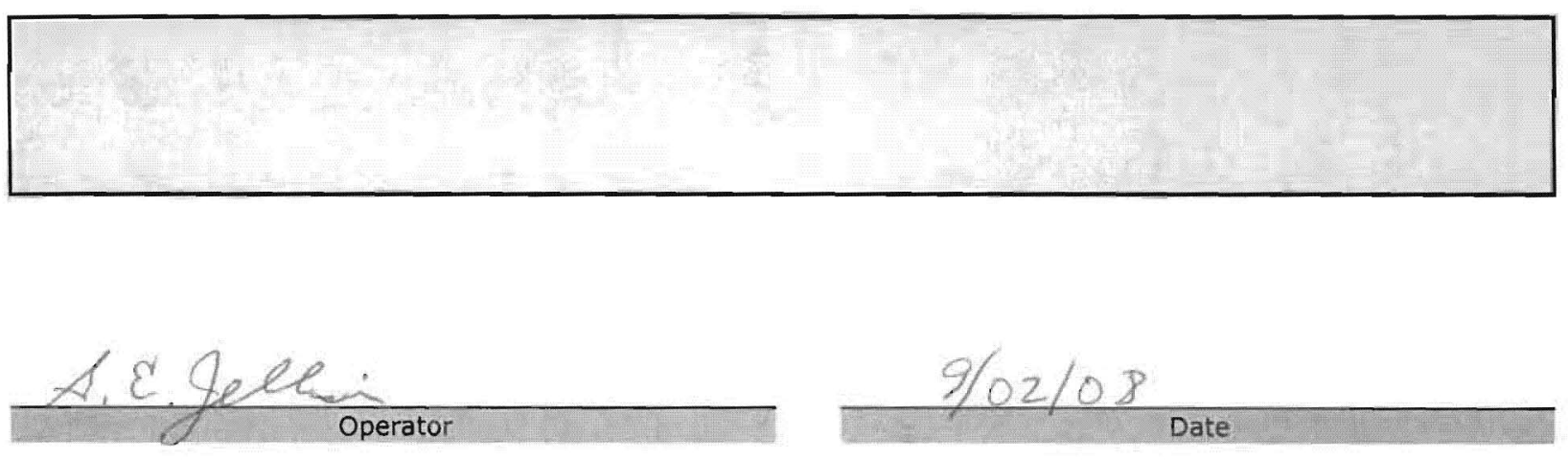


Data Report Form DRF-18A: Measurement of Pyrocarbon Anisotropy using the 2-MGEM - IPyC

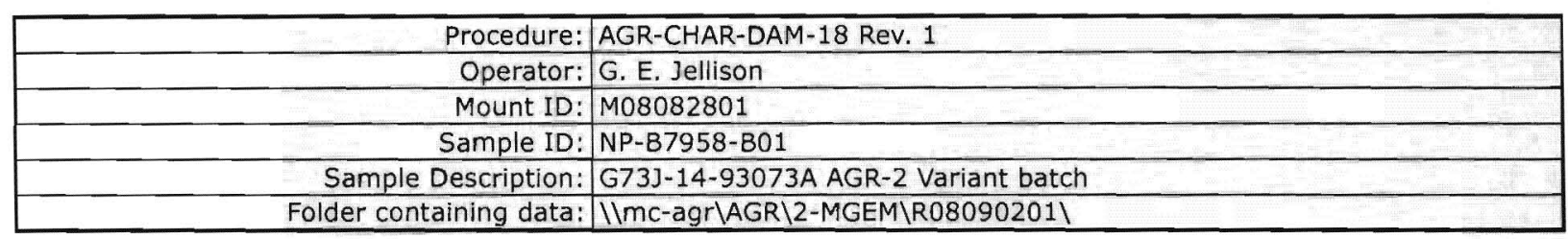

\begin{tabular}{|c|c|c|c|c|c|c|c|}
\hline \multirow{2}{*}{ Particle \# } & \multirow{2}{*}{$\begin{array}{c}\text { Grid } \\
\text { Position }\end{array}$} & \multicolumn{3}{|c|}{ Diattenuation } & \multicolumn{3}{|c|}{ True $B A F O=(1+N) /(1-N)$} \\
\hline & & Average & St. Dev. & Ave. Error & Average & St. Dev. & Ave. Error \\
\hline 1 & 4,4 & 0.0122 & 0.0018 & 0.0005 & 1.0247 & 0.0037 & 0.0010 \\
\hline 2 & 4,5 & 0.0117 & 0.0018 & 0.0005 & 1.0237 & 0.0037 & 0.0010 \\
\hline 3 & 4,6 & 0.0113 & 0.0021 & 0.0005 & 1.0229 & 0.0043 & 0.0010 \\
\hline 4 & 5,4 & 0.0116 & 0.0020 & 0.0005 & 1.0235 & 0.0041 & 0.0010 \\
\hline 5 & 5,5 & 0.0116 & 0.0021 & 0.0005 & 1.0235 & 0.0043 & 0.0010 \\
\hline 6 & 5,6 & 0.0112 & 0.0021 & 0.0005 & 1.0227 & 0.0043 & 0.0010 \\
\hline 7 & 6,4 & 0.0121 & 0.0020 & 0.0005 & 1.0245 & 0.0041 & 0.0010 \\
\hline 8 & 6,5 & 0.0118 & 0.0020 & 0.0005 & 1.0239 & 0.0041 & 0.0010 \\
\hline 9 & 6,6 & 0.0120 & 0.0018 & 0.0005 & 1.0243 & 0.0037 & 0.0010 \\
\hline 10 & 5,7 & 0.0109 & 0.0017 & 0.0005 & 1.0220 & 0.0035 & 0.0010 \\
\hline \multicolumn{2}{|c|}{ Average } & 0.0116 & 0.0019 & 0.0005 & 1.0236 & 0.0040 & 0.0010 \\
\hline
\end{tabular}

Mean of average BAFO per particle: 1.0236

Standard deviation of average BAFo per particle: 0.0008

\section{Comments}




\begin{tabular}{|c|c|}
\hline Procedure: & AGR-CHAR-DAM-18 Rev. 1 \\
\hline Operator: & G. E. Jellison \\
\hline Mount ID: & M08082801 \\
\hline Sample ID: & NP-B7958-B01 \\
\hline Sample Description: & G73J-14-93073A AGR-2 Variant batch \\
\hline Folder containing data: & IImc-agr\AGR\2-MGEM\R08090201\ \\
\hline
\end{tabular}

\begin{tabular}{|c|c|c|c|c|c|c|c|}
\hline \multirow{2}{*}{ Particle \# } & \multirow{2}{*}{$\begin{array}{c}\text { Grid } \\
\text { Position }\end{array}$} & \multicolumn{3}{|c|}{ Diattenuation } & \multicolumn{3}{|c|}{ True BAFo $=(1+N) /(1-N)$} \\
\hline & & Average & St. Dev. & Ave. Error & Average & St. Dev. & Ave. Error \\
\hline 1 & 4,4 & 0.0089 & 0.0016 & 0.0005 & 1.0180 & 0.0033 & 0.0010 \\
\hline 2 & 4,5 & 0.0090 & 0.0017 & 0.0005 & 1.0182 & 0.0035 & 0.0010 \\
\hline 3 & 4,6 & 0.0089 & 0.0018 & 0.0005 & 1.0180 & 0.0037 & 0.0010 \\
\hline 4 & 5,4 & 0.0092 & 0.0017 & 0.0005 & 1.0186 & 0.0035 & 0.0010 \\
\hline 5 & 5,5 & 0.0091 & 0.0020 & 0.0005 & 1.0184 & 0.0041 & 0.0010 \\
\hline 6 & 5,6 & 0.0082 & 0.0019 & 0.0005 & 1.0165 & 0.0039 & 0.0010 \\
\hline 7 & 6,4 & 0.0083 & 0.0016 & 0.0005 & 1.0167 & 0.0033 & 0.0010 \\
\hline 8 & 6,5 & 0.0082 & 0.0018 & 0.0005 & 1.0165 & 0.0037 & 0.0010 \\
\hline 9 & 6,6 & 0.0089 & 0.0018 & 0.0005 & 1.0180 & 0.0037 & 0.0010 \\
\hline 10 & 5,7 & 0.0089 & 0.0017 & 0.0005 & 1.0180 & 0.0035 & 0.0010 \\
\hline \multicolumn{2}{|c|}{ Average } & 0.0088 & 0.0018 & 0.0005 & 1.0177 & 0.0036 & 0.0010 \\
\hline
\end{tabular}

Mean of average BAFo per particle: 1.0177

Standard deviation of average BAFo per particle: 0.0008

\section{Comments}
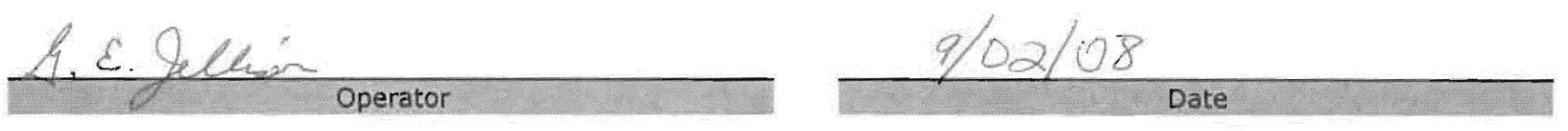
Data Report Form DRF-22: Estimation of Average Particle Welght

\begin{tabular}{|r|l|}
\hline Procedure: & AGR-CHAR-DAM-22 Rev. 1 \\
\hline Operator: & Dixie Barker \\
\hline Partlcle Lot ID: & NP-B7958 \\
\hline Particle Lot Description: & From G73J-14-93073A AGR-2 Variant Batch \\
\hline Fllename: & IIImC-agr|AGRIParticleWeightIW08082901_DRF22R1.x/s \\
\hline
\end{tabular}

\begin{tabular}{|r|c|c|c|c|c|}
\hline & Sample 1 & Sample 2 & Sample 3 & Sample 4 & Sample 5 \\
\hline Weight of particles (g): & 0.1593 & 0.1380 & 0.1353 & 0.1489 \\
\hline Number of particles: & 155 & 135 & 133 & 0.1203 \\
\hline Average welght/particle (g): & $1.028 \mathrm{E}-03$ & $1.022 \mathrm{E}-03$ & $1.017 \mathrm{E}-03$ & $1.027 \mathrm{E}-03$ & 117 \\
\hline
\end{tabular}

Mean average welght/particle $(g): 1.024 E-03$

Standard error in mean average welght/particle $(\mathrm{g}): 2.09 \mathrm{E}-06$
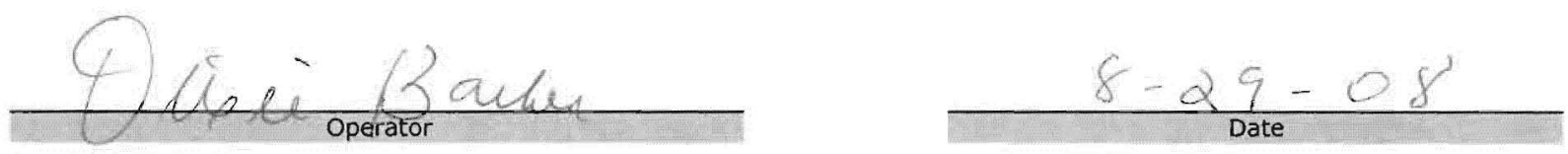


\section{Data Report Form DRF-31: Measurement of Open Porosity using a Mercury Porosimeter}

\begin{tabular}{|c|c|}
\hline Procedure: & AGR-CHAR-DAM-31 Rev. 1 \\
\hline Operator: & S. D. Nunn \\
\hline Coated particle batch ID: & NP-B7958-D01 \\
\hline Batch Description: & From G73J-14-93073A AGR-2 Variant batch \\
\hline Thermocouple Expiration Date: & $5 / 15 / 09$ \\
\hline Penetrometer Expiration Date: & $7 / 10 / 08$ \\
\hline Completed DRF Fllename: & IImc-agr|AGR\Poros/meter|S08091101/S08091101_DRF31R1.xIS \\
\hline
\end{tabular}

\begin{tabular}{|} 
Mean average weight/particle $(\mathrm{g}):$ & $1.02 \mathrm{E}-03$ \\
\hline Standard error in mean average weight/particle $(\mathrm{g}):$ & $3.87 \mathrm{E}-06$ \\
\hline Weight of particles $(\mathrm{g}):$ & 3.8113 \\
\hline ApproxImate number of particles: & 3722 \\
\hline Uncertainty in number of particles: & 14 \\
\hline Total envelope volume of sample $(\mathrm{cc}):$ & 1.276 \\
\hline Average envelope volume/particle $(\mathrm{cc}):$ & $3.43 \mathrm{E}-04$ \\
\hline Sample envelope density $(\mathrm{g} / \mathrm{cc}):$ & 2.988 \\
\hline Average particle dlameter $(\mathrm{microns}):$ & $8.68 \mathrm{E}+02$ \\
\hline Average surface area/particle $(\mathrm{cm} 2):$ & $2.37 \mathrm{E}-02$ \\
\hline Total sample surface area $(\mathrm{cm} 2):$ & $8.82 \mathrm{E}+01$ \\
\hline Open porosity $(\mathrm{ml} / \mathrm{m} 2):$ & $3.60 \mathrm{E}-03$ \\
\hline
\end{tabular}

\section{Comments}

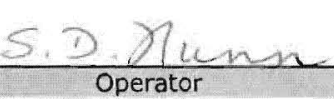


Data Report Form DRF-32: Counting of Particles with SIC Soot Inclusion Defects by Visual Inspection

\begin{tabular}{|c|c|}
\hline Procedure: & AGR-CHAR-DAM-32 Rev. 0 \\
\hline Operator: & Fred Montgomery \\
\hline Sample ID: & NP-B7958-E01 \\
\hline Sample Description: & From G73]-14-93073A AGR-2 Variant Batch \\
\hline Folder containing images: & 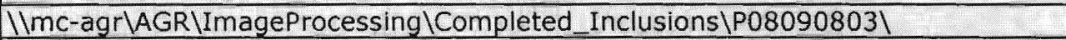 \\
\hline DRF filename: & 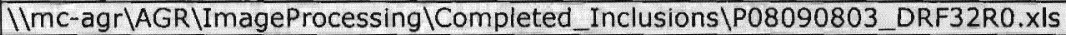 \\
\hline
\end{tabular}

\begin{tabular}{|r|l|}
\hline Mean average weight/particle $(\mathrm{g}):$ & $1.02 \mathrm{E}-03$ \\
\hline Uncertainty in average weight/particle $(\mathrm{g}):$ & $2.09 \mathrm{E}-06$ \\
\hline Weight of sample of particles $(\mathrm{g}):$ & 4.842 \\
\hline Approximate number of particles in sample: & 4729 \\
\hline Uncertainty in number of particles in sample: & 10 \\
\hline
\end{tabular}

Number of particles with $\mathrm{SiC}$ soot inclusion defects: 1

\section{Comments}

$1 / 4729$ corresponds to $<1 \mathrm{E}-3$ defect fraction at $95 \%$ confidence.

The one defect identified looked like it may be a chip in the SiC at the IPyC interface. Initial polish on mount 4 resulted in 5 particles with SiC gouges. These were removed by additional polishing, except for the deep one at the IPyC/SiC interface, which was identified as a defect.

Overall, the SiC/OPyC surface looks rough and is probably a strong interface.

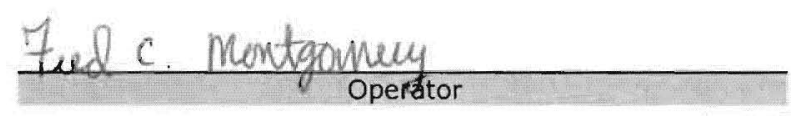

Date


From frame 43, mount 4 of NP-B7958-E01, G73J-14-93073A AGR-2 Variant batch.

This is the only particle out of 4729 identified with a bright field anomaly in the SiC. The defect appears to be a relatively large and deep chip at the IPyC interface. This may be related to a weakened region caused by a soot inclusion. However, normally a soot inclusion will extend over a greater arc.

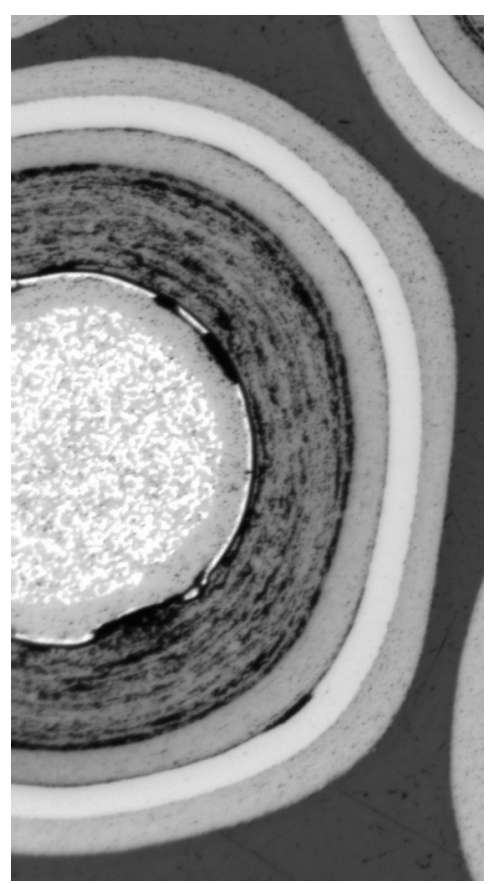

